\title{
Fever, mucocutaneous haemorrhage, and severe headache during an epidemic of haemorrhagic fever
}

\author{
A B Dey, D Chaudhury, A K Mohapatra, Kalpana M Nagarkar, O P Malhotra
}

A 36-year-old man developed moderate-grade fever, headache, and malaise. On the third day, he noticed purpuric skin lesions and sought medical opinion. Investigations revealed a platelet count of $57 \times 10^{9} / 1$ and haematocrit of $64 \%$. Several people in his locality and the city had similar illness with reported deaths. A diagnosis was made and he was treated with antipyretics for the next 3 days when he developed epistaxis, intense headache, and drowsiness. Cranial computed tomography (CT) (figures 1 and 2) was done and he was referred to this hospital for further management.

On admission he had active epistaxis and complained of persistent headache. History of head trauma in any form was denied. He had no history of any coexisting medical illness. He was febrile, drowsy but oriented, and co-operative. His blood pressure was $130 / 75 \mathrm{mmHg}$. He had left-sided hemiparesis (grade $4 / 5$ power) with brisk reflexes. There were no signs of meningeal irritation. Systemic examination did not reveal any abnormality. Laboratory investigations revealed a haemoglobin level of $8.9 \mathrm{~g} / \mathrm{dl}$, total leucocyte count was $7.9 \times 10^{9} / 1$ with a normal differential count, haematocrit $57 \%$ and platelet count $70 \times 10^{9} / 1$. Blood biochemical parameters and chest X-ray were normal. $\mathrm{He}$ was treated with an infusion of platelet-rich pląsma, and acetazolamide and mannitol to reduce cerebral oedema. Over the next 4 days, he became afebrile with a rise in platelet count to $130 \times 10^{9} / 1$. However, his neurological status deteriorated with increasing drowsiness, increase in left hemiparesis and bilateral extensor plantar response. A repeat cranial CT scan was not significantly different from the earlier one. In view of his neurological deterioration, he was subjected to a definitive procedure following which he steadily improved. Six months later he was doing well.

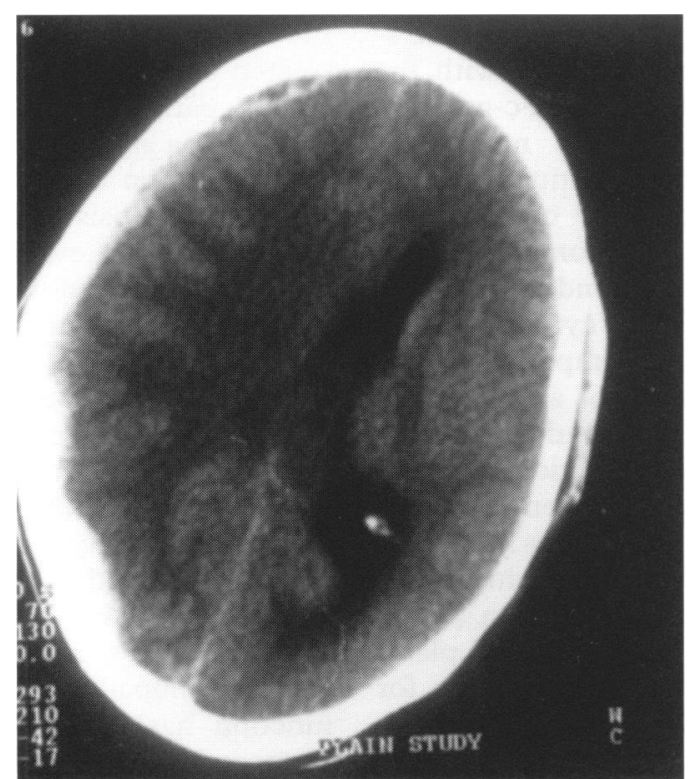

Figure 1 - Non-contrast cranial CT scan, axial cut

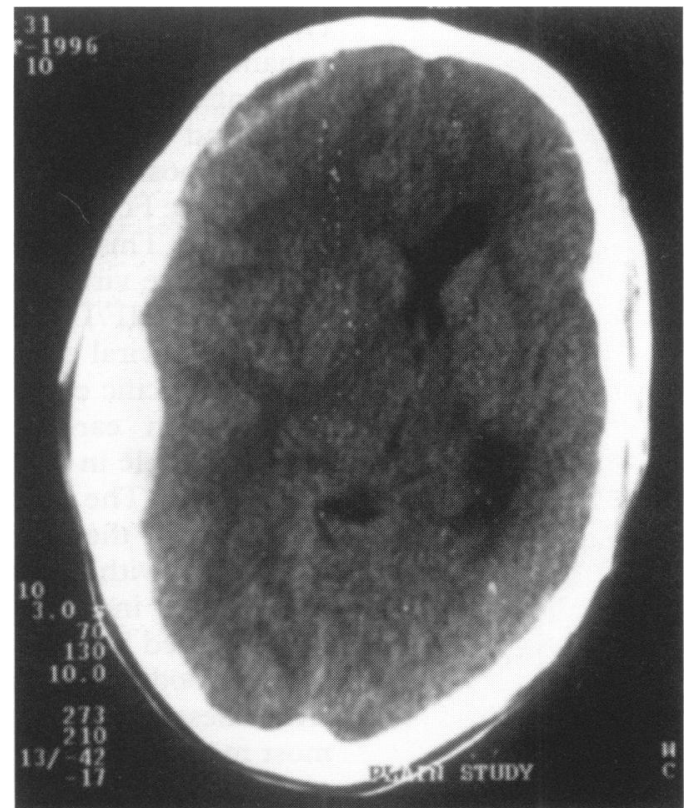

Figure 2 Non-contrast cranial CT scan, axial cut (higher cut)
Ansari Nagar,

New Delhi 110029,

India

Department of

Medicine

A B Dey

D Chaudhury

K M Nagarkar

O P Malhotra

Department of

Neurosurgery

A K Mohapatra

Accepted 21 January 1998

2 What was the primary disease? What are the criteria for its diagnosis, both in epidemics and definitive diagnosis?

3 What is the pathogenesis in this condition?

4 What are the causes of non-traumatic intracranial haemorrhage? 


\section{Answers}

QUESTION 1

The non-contrast cranial CT scan, axial cut, shows right-sided fronto-parietal subdural haematoma with midline shift, shifting of right choroid plexus anteriorly and medially, and complete obliteration of right lateral ventricle. The patient was subjected to a trephine craniotomy and evacuation of the haematoma.

\section{QUESTION 2}

The primary disease was dengue haemorrhagic fever (DHF). The World Health Organisation (WHO) definition criteria for DHF during epidemics include fever, muco-cutaneous bleeding, thrombocytopenia, and haemoconcentration. ${ }^{1} \mathrm{~A}$ definitive laboratory diagnosis requires virus isolation or serology. Polymerase-chainreaction-based diagnosis of the virus has also emerged as a reliable tool for diagnosis, although its routine use has yet to be evaluated.

QUESTION 3

The pathogenesis of severe complications of dengue virus infections is not clear. Dengue virus infection confers lifetime immunity against re-infection with homologous serotypes, while increasing the susceptibility to other serotypes with a higher risk of complications, such as DHF and dengue shock syndrome (DSS). Studies from Thailand have demonstrated that $90 \%$ of all DHF/DSS cases occur with secondary dengue infection. Increased susceptibility to a heterologous serotype is mediated by antibody-dependen enhancement, ie, sub-neutralising concentrations of antibodies form complexes with the virus and thereby bind to the Fc-gammareceptor-positive cells such as monocytes through the $\mathrm{Fc}$ portion of the immunoglobulin molecule. This enhancement of infection increases the virus load and promotes pathogenesis of DHF/DSS. The secondary antibody response to viral antigens and cytotoxic effect of virus-specific cytotoxic T-lymphocytes sensitised from earlier infection also has an important role in the immunopathogenesis of $\mathrm{DHF} / \mathrm{DSS}$. The immune reaction involves activation of the classical complement pathway along with release of various cytokines resulting in increased vascular permeability, disseminated intravascular coagulation (DIC) and antibody-mediated destruction of platelets. These three features are responsible for most manifestations of DHF/DSS.

QUESTION 4

The common causes of non-traumatic intracranial haemorrhage are given in box 1 .

\section{Discussion}

Acute subdural haematoma usually results from head trauma. Non-traumatic (spontaneous) subdural haematoma is a rare form of intracranial bleeding. Risk factors for spontaneous subdural haematoma are hypertension ${ }^{2}$ and bleeding diathesis secondary to DIC due to cancer, ${ }^{3}$ cirrhosis of liver ${ }^{4}$ and anticoagulant
Common causes of non-traumatic intracranial haemorrhage

- spontaneous intracerebral haemorrhage:

hypertension, amyloid angiopathy

- ruptured aneurysm

- ruptured arterio-venous malformation

- bleeding from primary and secondary tumours

- systemic bleeding disorders

- anticoagulation therapy

- drugs: cocaine, amphetamines

- haemorrhagic infarction

Box 1

\section{Learning points \\ - hypertension is the commonest cause of all types of spontaneous intracranial haemorrhage \\ - non-traumatic (spontaneous) subdural haematoma is extremely uncommon \\ - viral haemorrhagic fevers can be associated with intracranial haemorrhage}

Box 2

medication. ${ }^{5}$ Non-traumatic subdural haematoma has also been reported in association with dural metastasis ${ }^{6}$ and tumour embolism. The source of bleeding in two-thirds of cases is arterial. ${ }^{2}$ The interval between start of symptoms and diagnosis is variable from 1 day to 5 weeks. The condition has a variable prognosis depending on the size of the haematoma and the intervention provided. Most patients treated in time surgically survive. ${ }^{2}$

Our patient had non-traumatic subdural haematoma and satisfied the WHO criteria for case definition of DHF during epidemics. ${ }^{1}$ A definite diagnosis based on serology or viral isolation was not available in this patient, however serologic studies during the epidemic established dengue virus type II as the aetiologic agent. The haemorrhagic manifestations of DHF include spontaneous mucocutaneous haemorrhage, namely petechiae, ecchymosis, oozing from venepuncture sites, epistaxis and haematuria. Life-threatening gastrointestinal bleeding occurs in severely ill patients. The pathogenesis of the haemorrhagic manifestations in DHF is immune mediated and involves vascular injury, thrombocytopenia and coagulopathy. ${ }^{18}$ Qualitative platelet dysfunction has also been described in $\mathrm{DHF}^{8}$ Intracerebral and other forms of intracranial bleeding have not been reported in DHF or, for that matter, in any other type of viral haemorrhagic fever. The pathogenesis of subdural haematoma in the present case is similar to that due to $\mathrm{DIC}^{3}$ or anticoagulant medication, ${ }^{4}$ and represents a hitherto unreported complication of DHF.

\section{Final diagnosis}

Non-traumatic (spontaneous) subdural haematoma as a complication of dengue haemorrhagic fever. 
Keywords: subdural haematoma; dengue haemorrhagic fever; haemorrhage

1 World Health Organisation. Dengue hemorrhagic fever: diagnosis, treatment and control. Geneva: World Health Organisation, 1986.

2 Sawaya R, Means ED. Acute spontaneous subdural hematoma. Surg Neurol 1984;21:363-6.

3 Hanakita J, Kondo A, Nishihara K, Yamamoto Y, Kinuta Y Spontaneous subdural hematoma in patients with advanced cancer report of two cases. Neurol Med Chir Tokyo 1985;25: 568-70.

4 Yanai Y, Kohno N, Mitsui T. Acute spontaneous subdural hematoma of arterial origin. Surg Neurol 1985;23:417-20.
5 Kanter R, Kanter M, Krisch W, Rosenberg G. Spontaneous posterior fossa subdural hematoma as a complication of anticoagulation. Neurosurgery 1984;15:241-2.

6 Bucci MN, Farhat SM. Metastatic adenocarcinoma of the prostate as a cause of subdural hematoma. $\mathcal{F}$ Urol 1986;135: 803-4.

7 Cave WS. Acute non-traumatic subdural hematoma of arterial origin. F Forensic Sci 1983;28:786-9.

8 Halstead SB. Dengue: hematological aspects. Semin Hematol 1982;19:116-9.

\title{
An unusual soft tissue tumour and peripheral eosinophilia
}

\author{
B Banerjee, M Nashi, G R Dixon
}

A 34-year-old woman presented with a painless swelling over her left deltoid region of about 3 weeks duration. There was no history of injury, recent infection or vaccination. The patient occasionally suffered from hay-fever and low-grade joint pain. Clinical examination revealed a firm ill-defined $10 \mathrm{~cm} \times 8 \mathrm{~cm}$ non-tender swelling over the posterior aspect of the left deltoid muscle without induration of the overlying skin. No restriction of joint movements was noted.

Initial laboratory studies revealed a normal total white cell count but an elevated eosinophilic count of $12 \%$. Erythrocyte sedimentation rate (ESR) was $19 \mathrm{~mm} / \mathrm{h}$. Serum biochemical profile was within normal limits. Rheumatoid factor and antinuclear antibodies were negative. Plain radiograph of the left shoulder only demonstrated soft tissue thickening over the deltoid region. Computed tomography (CT) of the left shoulder is shown in figure 1 .
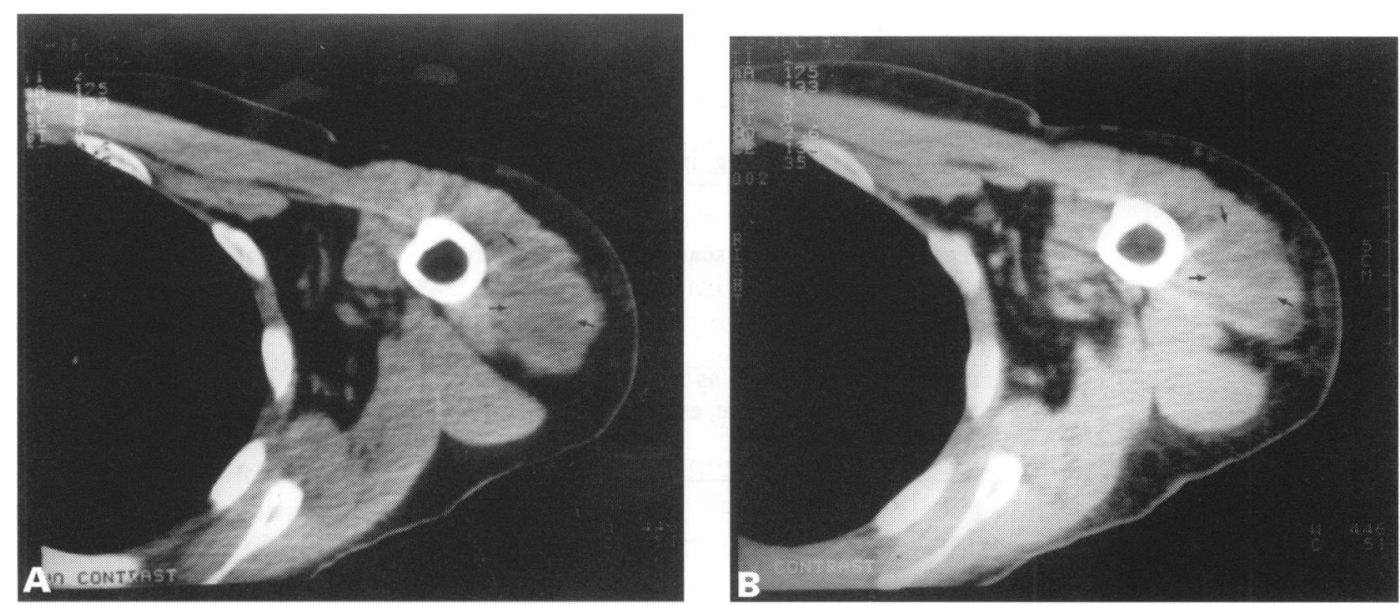

Figure 1 CT scans of the left shoulder, without (A) and with (B) intravenous contrast enhancement

Department of

Radiodiagnosis

B Banerjee

Department of

Orthopaedic Surgery

M Nashi

Department of

Pathology

G R Dixon

Accepted 21 January 1998

\section{Questions}

1 What do the CT scans show?

2 What is the most likely diagnosis?

3 What further investigation is required to confirm the diagnosis?

4 How is the condition managed? 